\title{
Proceedings of the 2017 ISNR Conference: Plenary
}

\section{Presentations}

\author{
Selected Abstracts of Conference Presentations at the 2017 International Society for Neurofeedback \\ and Research (ISNR) 25th Conference, Mashantucket, Connecticut, USA
}

Citation: International Society for Neurofeedback and Research. (2017). Proceedings of the 2017 ISNR conference: Plenary presentations. NeuroRegulation, 4(3-4), 146-157. http://dx.doi.org/10.15540/nr.4.3-4.146

Copyright: ( ) 2017. ISNR. This is an Open Access article distributed under the terms of the Creative Commons Attribution License (CC-BY).

Re-training the Injured Brain: sLORETA

Neurofeedback as an Acute Concussion

Intervention

David $I m s^{1,2}$

${ }^{1}$ Towson University, Towson, Maryland, USA

${ }^{2}$ Chesapeake Neurology Associates, Prince Frederick, Maryland, USA

Introduction/Support. Concussion incidence rates are at epidemiological levels and rising (Giza \& Hovda, 2001). In 2011, the Center for Disease Control (CDC) estimated 1.6-3.8 million sports- or recreation-related concussions occur per year (Daneshvar, Nowinski, McKee, \& Cantu, 2011), and concussion rates are increasing in adolescents (Zhang, Sling, Rugg, Feeley, \& Senter, 2016). Concussion is a complex pathophysiologic process affecting the brain, induced by biomechanical forces (McCrory et al., 2013). Concussion-related symptoms interrupt the daily functioning of the concussed individual, and current concussion recovery guidelines emphasize rest and the avoidance of symptom-provoking behaviors to minimize concussion-related symptoms throughout recovery (McCrory et al., 2013). Concussion injury results in measurable EEG abnormalities detectable by electrophysiological techniques (Rapp et al., 2015). Cortical deregulation due to concussion injury as depicted by qEEG may be addressed by neurofeedback shortly after injury.

Hypothesis/Justification. Neurofeedback has been used as an intervention for traumatic brain injury, but requires further investigation as an evidence-based practice. Neurofeedback is currently ranked as "Level 3 - Probably Efficacious" by the statement of efficacy on evidence-based practice in biofeedback and neurofeedback by the Association for Applied Psychophysiology and
Biofeedback (AAPB; Yucha \& Gilbert, 2004). The principle investigator hypothesizes that (1) neurofeedback interventions to inhibit slow wave activity will reduce the presence of concussion related symptoms and (2) the use of the intervention will decrease recovery time compared to recommended clinical guidelines of return-to-play concussion recovery.

Methods. As soon as logistically possible, qEEG data will be collected from adolescent or young adult patients presenting autonomously to a neurology clinic for the evaluation and treatment of acute concussion related to sport or recreational activity. Cognitive measurements and concussion-related symptoms will be measured and tracked using the XLNTbrain Sport Inc. Concussion Management Program as patients recover according to current evidence-based guidelines outlined by McCrory et al. (2013) qEEG data, neurocognitive test results, concussion-related symptoms, and attending neurologist who will guide neurofeedback protocol design. Data will be recollected after patients undergo approximately 15 sessions of neurofeedback and are medically cleared of concussion injury.

Results. Surface (scalp), sLORETA, and connectivity Z-score qEEG metrics from multiple commercially available qEEG database suites will be assessed for change from pre-neurofeedback and post-neurofeedback qEEG assessments. Changes in deviant SLORETA Z-scores and affected brain volume will be emphasized. Images of sLORETA results will be displayed visually and graphed by frequency band. Cognitive scores and concussionrelated symptoms following injury and after recovery will be assessed. 
Conclusion. Neurofeedback may provide a novel treatment option for the pervasive neuropsychological concussion problem. Findings that yield Z-scored brain activity largely approaching a statistically normal range $(Z=0)$ would suggest that neurofeedback may be beneficial to concussion recovery in comparison to recovery without the use of neurofeedback, where Z-scores may remain statistically deviant upon recovery from concussion injury (Ims, 2016). Theoretically, interventional neurofeedback following concussion injury may enable the concussed individual to train his or her brain towards recovery and alter the long-term trajectory of the injured brain.

\section{References}

Daneshvar, D. H., Nowinski, C. J., McKee, A. C., \& Cantu, R. C. (2011). The epidemology of sport-related concussion. Clinics in Sports Medicine, 30(1), 1-17. http://dx.doi.org/10.1016 /j.csm.2010.08.006

Giza, C. C., \& Hovda, D. A. (2001). The neurometabolic cascade of concussion. Journal of Athletic Training, 36(3), 228-235.

Ims, P. D. (October, 2016). Riding the Wave to Recovery: sLORETA QEEG in Sport-Related Concussions. Paper presented at the 24th Annual Conference of the International Society of Neurofeedback Research, Orlando, FL.

McCrory, P., Meeuwisse, W. H., Aubry, M., Cantu, B., Dvořák, J., Echemendia, R. J., ... Turner, M. (2013). Consensus statement on concussion in sport: The 4th International Conference on Concussion in Sport held in Zurich, November 2012. British Journal of Sports Medicine, 47(5), 250-258. http://dx.doi.org/10.1136/bjsports-2013-092313

Rapp, P. E., Keyser, D. O., Albano, A., Hernandez, R., Gibson, D. B., Zambon, R. A., ... Nichols, A. S. (2015). Traumatic brain injury detection using electrophysiological methods. Frontiers in Human Neuroscience, 9, 11. http://dx.doi.org/10.3389 /fnhum.2015.00011

Yucha, C., \& Gilbert, C. (2004). Evidence-Based Practice in Biofeedback and Neurofeedback. Wheat Ridge, CO: Association for Applied Psychophysiology and Biofeedback.

Zhang, A. L., Sling, D. C., Rugg, C. M., Feeley, B. T., \& Senter, C. (2016). The Rise of Concussions in the Adolescent Population. Orthopaedic Journal of Sports Medicine, 4(8). http://dx.doi.org/10.1177/2325967116662458

\section{ISNR FOUNDATIONS - Introduction to qEEG \\ Concepts and Applications}

Thomas Collura

The Brain Enrichment Center, Bedford, Ohio, USA

This oral presentation will provide an overview of the use of quantitative EEG (qEEG) in clinical practice. Emphasis will be placed on mental health applications. The basic functions of the human brain in relation to EEG will be described. Specific EEG components (frequency bands) will be explained, as well as the anatomical basis of EEG rhythms. Material will be drawn from published articles and books, and will emphasize current knowledge. The relationships of EEG amplitude and phase, connectivity, and brain activation will be described. From a basic knowledge of physiology and anatomy, the relevant clinical signs and symptoms can be put into context and used to create treatment planning. The use of normative reference databases will be explained, as well as relevant inclusion and exclusion criteria for creating clinically relevant databases. Signal processing concepts will also be introduced, including frequency analysis, use of summary maps and other graphical tools, and zscores. The relationship between EEG, qEEG, and neurofeedback will be explained in detail and put into a clinical framework that can be applied by diverse practitioners. The historical, scientific, and medical background of these topics will also be described, and specific examples of key research and clinical activities will be presented. In order to ensure a foundational approach, the basic theory of neuronal electrophysiology, volume conduction, EEG amplification, and signal processing will be described at a level accessible to mental health and medical practitioners. Relevant specifications and performance criteria for instrumentation and software will also be described, providing a grasp of the capabilities and limits of modern technology. A variety of clinical case studies will be used to illustrate key concepts and to demonstrate the utility of these approaches in the medical and mental health practice areas. The importance of recognizing individual differences, peak performance traits, and coping and compensatory mechanisms will also be covered, including the concepts of phenotypes and individual brain optimization strategies.

\section{References}

Collura, T. F. (2014). Technical Foundations of Neurofeedback. New York, NY: Routledge/Taylor \& Francis.

Collura, T. F., \& Frederick, J. A. (2016). Handbook of Clinical QEEG and Neurotherapy. New York, NY: Taylor \& Francis.

Niedermeyer, E. (2012). Clinical Electroencephalography. New York, NY: Lippincott.

Thatcher, R. W. (2012). Handbook of Quantitative Electroencephalography and EEG Biofeedback. St. Petersburg, FL: ANI Publishing. 


\author{
A Model for qEEG, and sLORETA Correlates to \\ Predicting and Enhancing Human \\ Performance: A Multivariate Approach \\ David Cantor ${ }^{1}$, Dick Genardi ${ }^{2}$, Barbara Minton ${ }^{3}$, and \\ Robert Chabot ${ }^{4}$ \\ ${ }^{1}$ Mind and Motion Developmental Centers of Georgia, Johns \\ Creek, Georgia, USA \\ ${ }^{2}$ Licensed Clinical Psychologist, Centerville, Ohio, USA \\ ${ }^{3}$ Licensed Clinical Psychologist, Boise, Idaho, USA \\ ${ }^{4}$ BrainDx, Johns Creek, Georgia, USA
}

The derivation of $\mathrm{qEEG}$ predictive functions may play a critical role in using qEEG measures that predict potential functional weaknesses or individuals "at risk" for specific academic or occupational challenges. Such measures can also hypothetically be used in a multivariate algorithm approach in neurofeedback to achieve human performance enhancement. EEG was obtained from 493 individuals ranging in age from 4 to 75 years diagnosed with a variety of disorders. All EEG data used a 19-site monopolar montage and referenced to $\mathrm{Cz}$ in acquisition and utilizing linked ears reference to derive digitized information in each of the following broad bands: delta $(1.5-3.5 \mathrm{~Hz})$, theta (4.0-7.5 Hz), alpha $(8.0-12.5 \mathrm{~Hz})$, beta (13.0-25.0 $\mathrm{Hz})$, and beta2 $(25.5-35.0 \mathrm{~Hz})$ frequency bands with derived measures of absolute power, relative power, power asymmetry (inter- and intrahemispheric), coherence (inter- and intrahemispheric), and mean frequency for each broad band was attained and then converted to $z$-scores relative to a database of age-matched normal. Univariate as well as complex multivariate variables collapsed across selected regions and combination of frequencies were derived for a total of 13,712 variables. Additionally, the sLORETA voxels including weighted function voxels for subcortical structures were derived at very narrow band frequencies $(.39 \mathrm{~Hz}$ bands) ranging from $1.5 \mathrm{~Hz}$ to $35.5 \mathrm{~Hz}$ of the EEG (87 variables). Zscores of all voxels for each ROI standard in sLORETA as well as a number of weighted voxels estimating subcortical locations were derived for each very narrow band frequency for a total of 6,896 variables. Data reduction methods for this total 599,952 variable matrix were utilized by deriving the mean Z-score score of all voxels within each ROI and then averaging these mean Z-scores across the narrow band frequencies that define each of a number of broad band frequencies for each subject. Step-wise regression analyses of the resultant reduced variable sets were used to define specific weighted polynomial multivariate equations accounting for over $90 \%$ of variance that predict standard scores from neuropsychological tests and their subtests for many cognitive and behavior measures. Analyses revealed distinctive predictive equations for human performance spanning a wide range of human performance. It is proposed that these algorithms represent electrophysiological base networks (as opposed to fMRI based networks) at "resting state" that correspond to gradients of psychological performance. Pilot data from equations demonstrated predictive ability to test neuropsychological performance were tested in independent patient samples to test validity and reliability. This study demonstrates that qEEG can be used to screen for brain functional impairment with prediction of specific neuropsychological deficits that may require further assessment and intervention. A discussion will be provided regarding the use of these same algorithms for neurofeedback training optimized human performance.

\section{References}

John, E. R., Prichep, L. S. (2006). The relevance of QEEG to the evaluation of behavioral disorders and pharmacological interventions. Clinical EEG and Neuroscience, 37(2),135-43. http://dx.doi.org/10.1177/155005940603700210

Johnstone, J., Gunkelman, J., \& Lunt, J. (2005). Clinical database development: Characterization of EEG phenotypes. Clinical EEG and Neuroscience, 36(2), 99-107. http://dx.doi.org/10.1177/155005940503600209

Pascual-Marqui, R. D., Esslen, M., Kochi, K., \& Lehmann, D. (2002). Functional imaging with low-resolution brain electromagnetic tomography (LORETA): Review, new comparisons, and new validation. Japanese Journal of Clinical Neurophysiology, 30, 81-94.

Reisberg, B., Prichep, L., Mosconi, L., John, E. R., GlodzikSobanska, L., Boksay, ... de Leon M. J. (2008). The pre-mild cognitive impairment, subjective cognitive impairment stage of Alzheimer's disease. Alzheimer's \& Dementia, 4(1, Suppl. 1), S98-S108. http://dx.doi.org/10.1016/j.jalz.2007.11.017

Thatcher, R. W. (2015). Handbook of Quantitative Electroencephalography and EEG Biofeedback, ebook Edition 1.1.2. St. Petersburg, FL: ANI Publishing.

\section{ISNR FOUNDATIONS - How Accurate}

\section{Assessment Leads to Effective Intervention} for ASD

Robert Coben and Anne Stevens

Integrated Neuroscience Services, Fayetteville, Arkansas, USA

Autism Spectrum Disorder (ASD) can be described as a "group of developmental disabilities that can cause significant social, communication, and behavioral challenges." The epidemiological data presented by the CDC reported that 1 in 68 children are currently diagnosed with ASD (CDC, 2016). The CDC has noted an increase in the prevalence of ASD since the 1990s, making effective treatment an important part of the growing conversation. Providing effective treatment options is becoming more and more crucial for this population, especially when considering the cost of healthcare for these 
individuals. With rates of incidence rising, this condition has become a major healthcare crisis calling for effective methods of intervention and an intimate understanding of the disorder. Neurofeedback is one promising method to treat the symptoms of ASD (Coben, 2013). This presentation will focus on providing up to date information on the clinical, neurophysiological, and neuropsychological underpinnings for individuals with Autism Spectrum Disorders (ASD) and how to use this information for better treatment plans and improved outcomes.

This presentation will discuss the importance of considering ASD in the context of underlying neurophysiological and neurocognitive correlates. When considering a treatment plan, this information can be used to rate the baseline symptoms in objective manner, as well as objectively measure change through the treatment course. This approach also allows the clinician more information to use to make adjustments during the treatment process. It is apparent that an optimal treatment course will demonstrate measurable change at the subjective, neurophysiological, and neurocognitive/ behavioral levels of analysis.

This presentation will outline assessment opportunities for various mental health professionals to consider adding to their own practice. Further discussion will relate this information to the EEG analysis. Next, steps will be provided regarding using the information for establishing the neurofeedback protocol. The presentation will provide a basic foundation for assessment opportunities for the neurofeedback clinician and strategies on how to use this information to make a more informed choice about neurofeedback treatment protocols. Using this information to measure outcome goals will also be discussed. Case presentations will also be provided to further a practical understanding.

\section{References}

Casanova, M. F., El-Baz, A. S., \& Suri, J. S. (2013). Imaging the Brain in Autism. New York, NY: Springer-Verlag.

Centers for Disease Control and Prevention. (2016). Autism spectrum disorder (ASD). Retrieved from http://www.cdc.gov /ncbddd/autism/index.html

Coben, R., \& Wagner, L. A. (2011). Emerging empirical evidence supporting connectivity-guided Neurofeedback for Autistic disorders. In R. Coben \& J. R. Evans (Eds.), Neurofeedback and Neuromodulation techniques and applications (pp. 153182). London, UK: Elsevier.

Rossignol, D. A., Rossignol, L. W., James, S. J., Melnyk, S., \& Mumper, E. (2007). The effects of hyperbaric oxygen therapy on oxidative stress, inflammation, and symptoms in children with autism: An open-label pilot study. BMC Pediatrics, 7, 3649. http://dx.doi.org/10.1186/1471-2431-7-36
An Analysis of Long-Term and Secondary

Outcomes of a Randomized Controlled Trial of

Neurofeedback to Treat Chemotherapy-

Induced Peripheral Neuropathy

Sarah Prinsloo ${ }^{1}$, Randall Lyle ${ }^{2}$, Diane Novy ${ }^{1}$, Larry

Driver ${ }^{1}$, Lois Ramondetta', Cathy Eng ${ }^{1}$, Gabriel Lopez ${ }^{1}$, Sunil Patel ${ }^{1}$, Jennifer McQuade ${ }^{1}$, and Lorenzo Cohen ${ }^{1}$

${ }^{1} \mathrm{MD}$ Anderson Cancer Center, Houston, Texas, USA

${ }^{2}$ Mount Mercy University, Cedar Rapids, lowa, USA

Background. CIPN is a common side effect of chemotherapy, leading to impairment in daily activities and diminished quality of life. Neurofeedback (NF) is a brain-training paradigm that induces neuroplasticity to modulate brain activity, and we have previously shown it leads to improved CIPN symptoms that were mediated by NF-induced brain changes.

Methods. Seventy-one (62 female; mean age $=63$; 52 breast, 8 gynecologic, 11 other; average length of symptoms $=24$ months) cancer survivors greater than three months from completing chemotherapy who reported greater than 3 on the NCl's neuropathy rating scale, were randomized to a NF group $(n=35)$ and underwent 20 sessions of electroencephalography (EEG) NF or a wait-list control group (WL; $n=36$ ). We used quantitative EEG imaging to determine any EEG patterns unique to CIPN and then provided NF to change aberrant brain signatures. The primary outcome measure was the Brief Pain Inventory (BPI). Secondary outcome measures included the Pain Quality Assessment Scale (PQAS), MD Anderson Symptom Inventory (MDASI), Short Form 36 (SF-36); Brief Fatigue Inventory (BFI); and Pittsburgh Sleep Quality Index (PSQI), which were completed at baseline, at the end of treatment, 1 and 4 months later. Analyses were done using a general linear mixed model regression (GLMM), and general linear regression (GLM) determined between group differences at each time point.

Results. $89 \%$ of the participants who were randomized completed treatment and $100 \%$ of participants who started NF completed treatment. Change scores from baseline to end of treatment demonstrate NF lead to significant reduction in neuropathic symptoms such as pain and numbness (previously reported), and in cancer-related symptom interference (NF $=-5.3$ vs $\mathrm{WL}=-0.5, p=$ .000 ); symptom severity (NF $=-5.1 \mathrm{vs} \mathrm{WL}=-0.8, p$ $=.000$ ); fatigue (NF $=-3.7$ vs $\mathrm{WL}=-0.8, p=.001)$, and sleep disturbances (NF $=-2.3$ vs $\mathrm{WL}=0.8, p=$ $.030)$; and improved physical functioning (NF $=3.3$ vs $\mathrm{WL}=1.4, p=.003$ ). At 4 months, the outcomes 
remained for targeted symptoms but not for secondary outcomes.

Conclusion. NF clinically and significantly improved primary outcomes at 4 months posttreatment and reduced secondary symptoms associated with CIPN.

\section{References}

Atkinson, T. M., Mendoza, T. R., Sit, L., Passik, S., Scher, H. I., Cleeland, C., \& Basch, E. (2010). The Brief Pain Inventory and its "Pain at its Worst in the last 24 Hours" Item: Clinical Trial Endpoint Considerations. Pain Medicine (Malden, Mass.), 11(3), 337-346. http://dx.doi.org/10.1111/j.15264637.2009.00774.x

Bao, T., Basal, C., Seluzicki, C., Li, S. Q., Seidman, A. D., \& Mao, J. J. (2016). Long-term chemotherapy-induced peripheral neuropathy among breast cancer survivors: Prevalence, risk factors, and fall risk. Breast Cancer Research and Treatment, 159(2), 327-333. http://dx.doi.org/10.1007/s10549-016-39390

Brami, C., Bao, T., \& Deng, G. (2016). Natural products and complementary therapies for chemotherapy-induced peripheral neuropathy: A systematic review. Critical Reviews in Oncology/Hematology, 98, 325-334. http://dx.doi.org /10.1016/j.critrevonc.2015.11.014

Cleeland, C. S., Mendoza, T. R., Wang, X. S., Chou, C., Harle, M. T., Morrissey, M., \& Engstrom, M. C. (2000). Assessing symptom distress in cancer: The M. D. Anderson Symptom Inventory. Cancer, 89(7), 1634-1646. http://dx.doi.org /10.1002/1097-0142(20001001)89:7<1634::AIDCNCR29>3.0.CO;2-V

Cleeland, C. S., \& Ryan, K. M. (1994). Pain assessment: Global use of the Brief Pain Inventory. Annals of the Academy of Medicine, Singapore, 23(2), 129-138.

Diers, M., Kamping, S., Kirsch, P., Rance, M., Bekrater-Bodmann, R., Foell, J., ... Maaß, H. (2015). Illusion-related brain activations: A new virtual reality mirror box system for use during functional magnetic resonance imaging. Brain Res, 1594, 173-182. http://dx.doi.org/10.1016 /j.brainres.2014.11.001

Jensen, M. P., Gammaitoni, A. R., Olaleye, D. O., Oleka, N., Nalamachu, S. R., \& Galer, B. S. (2006). The pain quality assessment scale: Assessment of pain quality in carpal tunnel syndrome. The Journal of Pain, 7(11), 823-832. http://dx.doi.org/10.1016/j.jpain.2006.04.003

Johnston, S. A., Ware, J. E., Jr., Davies-Avery, A., \& Brook, R. H. (1994). Conceptualization and measurement of health for adults in the Health Insurance Study (Mental Health R1987/3-HEW: Vol. 3). Santa Monica, CA: RAND Corporation.

Kinreich, S., Podlipsky, I., Jamshy, S., Intrator, N., \& Hendler, T. (2014). Neural dynamics necessary and sufficient for transition into pre-sleep induced by EEG neurofeedback. Neurolmage, 97, 19-28. http://dx.doi.org/10.1016 /j.neuroimage.2014.04.044

Kolb, N. A., Smith, A. G., Singleton, J. R., Beck, S. L., Stoddard, G. J., Brown, S., \& Mooney, K. (2016). The Association of Chemotherapy-Induced Peripheral Neuropathy Symptoms and the Risk of Falling. JAMA Neurology, 73(7), 860-866. http://dx.doi.org/10.1001/jamaneurol.2016.0383

Land, S. R., Kopec, J. A., Cecchini, R. S., Ganz, P. A., Wieand, H. S., Colangelo, L. H., \& Wolmark, N. (2007). Neurotoxicity from oxaliplatin combined with weekly bolus fluorouracil and leucovorin as surgical adjuvant chemotherapy for stage II and III colon cancer: NSABP C-07. Journal of Clinical Oncology, 25(16), 2205-2211. http://dx.doi.org/10.1200 /JCO.2006.08.6652
Majithia, N., Temkin, S. M., Ruddy, K. J., Beutler, A. S., Hershman, D. L., \& Loprinzi, C. L. (2016). National Cancer Institute-supported chemotherapy-induced peripheral neuropathy trials: Outcomes and lessons. Supportive Care in Cancer, 24(3), 1439-1447. http://dx.doi.org/10.1007/s00520015-3063-4

Mendoza, T. R., Wang, X. S., Cleeland, C. S, Morrissey, M., Johnson, B. A., Wendt, J. K., \& Huber, S. L. (1999). The rapid assessment of fatigue severity in cancer patients. Cancer, 85(5), 1186-1196. http://dx.doi.org/10.1002/(SICl)10970142(19990301)85:5\%3C1186::AID-CNCR24\%3E3.0.CO;2-N

Park, S. B., Lin, C. S. Y., Krishnan, A. V., Goldstein, D., Friedlander, M. L., \& Kiernan, M. C. (2011). Long-Term Neuropathy After Oxaliplatin Treatment: Challenging the Dictum of Reversibility. The Oncologist, 16(5), 708-716. http://dx.doi.org/10.1634/theoncologist.2010-0248

Prinsloo, S., Novy, D., Driver, L., Lyle, R., Ramondetta, L., Eng, C., ... Cohen, L. (2017). Randomized controlled trial of neurofeedback on chemotherapy-Induced peripheral neuropathy: A pilot study. Cancer, 123(11), 1989-1997. http://dx.doi.org/10.1002/cncr.30649

Rao, R. D., Michalak, J. C., Sloan, J. A., Loprinzi, C. L., Soori, G. S., Nikcevich, D. A., \& the North Central Cancer Treatment Group. (2007). Efficacy of gabapentin in the management of chemotherapy-induced peripheral neuropathy: a phase 3 randomized, double-blind, placebo-controlled, crossover trial (N00C3). Cancer, 110(9), 2110-2118. http://dx.doi.org /10.1002/cncr.23008

Seretny, M., Currie, G. L., Sena, E. S., Ramnarine, S., Grant, R., MacLeod, M. R., ... Fallon M. (2014). Incidence, prevalence, and predictors of chemotherapy-induced peripheral neuropathy: A systematic review and metaanalysis. Pain, 155(12), 2461-2470. http://dx.doi.org/10.1016 /j.pain.2014.09.020

Siegal, T., \& Haim, N. (1990). Cisplatin-induced peripheral neuropathy. Frequent off-therapy deterioration, demyelinating syndromes, and muscle cramps. Cancer, 66(6), 1117-1123. http://dx.doi.org/10.1002/10970142(19900915)66:6<1117::AIDCNCR2820660607>3.0.CO;2-O

Stubblefield, M. D., Burstein, H. J., Burton, A. W., Custodio, C. M., Deng, G. E., Ho, M., ... Von Roenn, J. H. (2009). NCCN task force report: Management of neuropathy in cancer. Journal of the National Comprehensive Cancer Network (JNCCN), 7(Suppl. 5), S1-S26; quiz, S27-S28. http://dx.doi.org /10.6004/jnccn.2009.0078

Surmeli, T., Eralp, E., Mustafazade, I., Kos, I. H., Özer, G. E., \& Surmeli, O. H. (2017). Quantitative EEG Neurometric Analysis-Guided Neurofeedback Treatment in Postconcussion Syndrome (PCS): Forty Cases. How Is Neurometric Analysis Important for the Treatment of PCS and as a Biomarker? Clinical EEG and neuroscience, 48(3), 217230. http://dx.doi.org/10.1177/1550059416654849

van Kerckhoven, G., Mert, A., \& De Ru, J. A. (2014). Treatment of vertigo and postural instability using visual illusions. The Journal of Laryngology \& Otoogyl, 128(11), 1005-1007. http://dx.doi.org/10.1017/S0022215114002254

Ware, J. E., Jr., Snow, K. K., Kosinski, M., \& Gandek, B. (1993). SF-36 Health Survey Manual and Interpretation Guide. Boston, MA: The Health Institute, New England Medical Center Hospitals, Inc.

Zhang, H., Li, Y., de Carvalho-Barbosa, M., Kavelaars, A., Heijnen, C. J., Albrecht, P. J., \& Dougherty, P. M. (2016). Dorsal root ganglion infiltration by macrophages contributes to paclitaxel chemotherapy-induced peripheral neuropathy. The Journal of Pain: Official Journal of the American Pain Society, 17(7), 775-786. http://dx.doi.org/10.1016 /j.jpain.2016.02.011 


\section{Depression Two Years Post Four-Channel Multivariate Coherence Neurofeedback Treatment}

Abby Bolen, Caitlinn Mosley, and Robert Coben Integrated Neuroscience Services, Fayetteville, Arkansas, USA

Having high prevalence and a persistent nature, it becomes clear that depression can be blighting for those struggling with its symptoms (Kessler \& Bromet, 2013). According to the World Health Organization, there are more than 300 million people worldwide who are suffering from depression (WHO, 2017). Many options for treatment, such as psychotherapy and medication, have been shown to be effective in reducing depression. However, a recent study by Stevens, Coben, and Middlebrooks (2015) showed that four-channel multivariate coherence neurofeedback treatment proved to be more effective in reducing depression compared to alternative methods. The current study aims to show that four-channel neurofeedback training is not only effective for the duration of treatment, but continues to maintain positive effects over time.

The original study mentioned consisted of 54 patients across three conditions: psychotherapy, neurofeedback, and a waitlist control group. The patients who did not receive neurofeedback initially were later offered neurofeedback treatment at the conclusion of the original study (crossover design). After completing the crossover study (Study 1), results showed that 15 out of the 18 patients who received NFB had significant changes over time, leading to a decrease in depression by 1 standard deviation, compared to the other conditions which did not show significance (Stevens, Coben, \& Middlebrooks, 2015). Our current sample consists of 48 patients involved in the original study who were administered the Beck Depression Inventory (BDI-II). In order to show the continued effects of neurofeedback on depression, the current follow-up study will compare the differences in depressive symptoms immediately following the completion of treatment and 2 years after the treatment has concluded. SPSS software will be used in order to perform an ANOVA for analysis. Results demonstrating the changes in reported symptoms are expected to support our hypothesis that fourchannel multivariate coherence training will maintain the reduction of depressive symptoms 2 years after the conclusion of treatment.

The data from the initial crossover study, as well as the current follow-up study, is expected to provide evidence that four-channel multivariate coherence neurofeedback treatment can have continuing positive effects for individuals struggling with depression. With the commonality of depression present today, it is important we continue to expand our knowledge and explore the most effective treatment options.

\section{References}

Beck, A. T., Steer, R. A., \& Brown, G. K. (1996). Manual for the Beck Depression Inventory-II (2nd ed.). San Antonio, TX: The Psychological Corporation.

Hammond, D. C. (2005). Neurofeedback treatment of depression and anxiety. Journal of Adult Development, 12(2-3), 131137. http://dx.doi.org/10.1007/s10804-005-7029-5

Kessler, R. C., \& Bromet, E. J. (2013). The epidemiology of depression across cultures. Annual Review of Public Health, 34, 119-138. http://dx.doi.org/10.1146/annurev-publhealth031912-114409

Khan, A., Faucett, J., Lichtenberg, P., Kirsch, I., \& Brown, W. A. (2012). A systematic review of comparative efficacy of treatments and controls for depression. PLOS ONE, 7(7), e41778. http://dx.doi.org/10.1371/journal.pone.0041778

Stevens, A., Coben, R., \& Middlebrooks, M. (2015). The Impact of Coherence on Depression. Lecture presented at Annual International Society for Neurofeedback and Research Conference in Denver, Colorado.

World Health Organization. (2017). Depression. Retrieved from http://www.who.int/mediacentre/factsheets/fs369/en/

The Use of Four-Channel Multivariate Training on Mild Traumatic Brain Injury: A Comparison of Newly Concussed and Remotely Concussed Individuals

Anne Stevens and Morgan Middlebrooks

Integrated Neuroscience Services, Fayetteville, Arkansas, USA

Mild traumatic brain injury (MTBI), commonly referred to as a concussion, can be described as a brain injury resulting from acute trauma to the head (Carroll, Cassidy, Holm, Kraus, \& Coronado, 2004). In the United States it is reported that up to 1.7 million people sustain a traumatic brain injury (TBI) annually, which has resulted in the direct and indirect cost of $\$ 60$ billion in the United Sates. However, it is estimated that the burden of TBI is dramatically underrepresented as many people who experience mild or moderate TBI do not seek medical support. With an estimated 38 million children involved in athletic activities, it is important to consider the effects that MTBI presents to the United States (Daneshvar, Nowinski, Mckee, \& Cantu, 2011). MTBI is commonly associated with fatigue, headaches, memory loss, poor attention, sleep disturbances, seizures, feelings of depression, and other significant symptoms (Grady, 2010).

The scope of MTBI makes improved detection along with ease and efficacy of treatment vital. Postconcussion $86 \%$ of patients are found to have 
abnormal EEG making EEG an important tool in the detection of MTBI. Additionally, the use of neuropsychological testing can help to identify the lasting effects of MTBI (Haneef, Levin, Frost, \& Mizrahi, 2013). When considering the types of improvements that different therapy modalities make, time of treatment has always been a point of discussion for treatment providers. Many physicians believe that the patient should engage in a period of rest before starting therapy to improve MTBI symptomology. We intend to explore the relationship between functional outcomes of the concussed patient and the amount of time elapsed before the start of treatment.

Methods. Our study aims to compare outcomes of four-channel coherence training with recent versus remotely concussed individuals using a case series methodology. Patients diagnosed with MTBI will undergo a qEEG to develop individual protocol for four-channel multivariate coherence training. Preand post-qEEG will be done to appreciate global changes in coherence. Additionally, other neuropsychological testing indices will be administered to provide a functional look at changes in MTBI-related symptomology. The correlation between demographic information and outcome of treatment will be explored as well.

Assessment. Quantitative electroencephalogram studies were performed pre- and posttreatment using Brain Dx, NeuroRep, and Neuroguide. Additionally, the use of multiple neuropsychological tests was employed.

Anticipated Results/Hypothesis. We anticipate that the use of four-channel multivariate coherence neurofeedback training will improve the functional outcome of the concussed patient, regardless of time of injury.

\section{References}

Carroll, L. J., Cassidy, J. D., Holm, L., Kraus, J., \& Coronado, V. G. (2004). Methodological issues and research recommendations for mild traumatic brain injury: The WHO collaborating centre task force on mild traumatic brain injury. Journal of Rehabilitation Medicine, 36(43), 113-125. http://dx.doi.org/10.1080/16501960410023877

Daneshvar, D. H., Nowinski, C. J., McKee, A. C., \& Cantu, R. C. (2011). The Epidemiology of Sport-Related Concussion. Clinics in Sports Medicine, 30(1), 1-17. http://dx.doi.org/10.1016/j.csm.2010.08.006

Grady, M. F. (2010). Concussion in the adolescent athlete. Current Problems in Pediatric and Adolescent Health Care, 40(7), $\quad 154-169 . \quad \mathrm{http}: / / \mathrm{dx}$.doi.org/10.1016 /j.cppeds.2010.06.002

Haneef, Z., Levin, H. S., Frost, J. D., \& Mizrahi, E. M. (2013). Electroencephalography and Quantitative
Electroencephalography in Mild Traumatic Brain Injury. Journal of Neurotrauma, 30(8), 653-656. http://dx.doi.org /10.1089/neu.2012.2585

Thompson, M., Thompson, L., Reid-Chung, A., \& Thompson, J. (2013). Managing Traumatic Brain Injury: Appropriate Assessment and a Rationale for Using Neurofeedback and Biofeedback to Enhance Recovery in Postconcussion Syndrome. Biofeedback, 41(4), 158-173. http://dx.doi.org/10.5298/1081-5937-41.4.07

\section{Enhancing Treatment Success: The Benefits of EEG Analysis}

Ronald Swatzyna, Laura Childers, and Erin Maclnerney Tarnow Center for Self-Management, Houston, Texas, USA

A large number of neurofeedback candidates have tried a multitude of approaches, treatment modalities, and medications with limited success. Studies find qEEG neurofeedback successful 60 to 80 percent of the time, leaving 20 to 40 percent unsuccessful. This sizable percentage of treatment failure could be avoided by having the electroencephalogram (EEG) analyzed by a electrophysiologist or neurologist who is board certified in electroencephalography. A recent EEG/qEEG study identified four neurobiomarkers that accounted for psychotropic medication failure: focal slowing, beta spindles, encephalopathy, and transient discharges (Swatzyna et al., 2014). Although the qEEG is excellent for identifying the location and significance of focal slowing as well as excessive beta, only the EEG can identify the morphology of beta spindles. In a study by Arns, Swatzyna, Gunkelman, and Olbrich (2015), beta spindles were found to be associated with sleep issues and were best evaluated with polysomnography. In addition, Swatzyna et al. (2015) found that individuals with beta spindles were five times more likely to be diagnosed with depression and experienced treatment resistance to selective serotonin reuptake inhibitors (Arns, 2011). Many sleep issues require a medical intervention and often account for treatment failure. A low voltage slow EEG is the hallmark of encephalopathy, but only an electroencephalographer can identify the associated morphology. Unless the brain has enough oxygenation or metabolic support, all therapies and medications will have limited effectiveness. Lastly, transient discharges are often averaged out or removed in the qEEG process. However, identifying the source of these discharges is often critical to protocol design and treatment success. Although everyone who utilizes qEEG collects EEG data, few clinicians have the data read by a board certified electroencephalographer. This presentation provides case examples where data from an EEG provided critical information such as 
structural, metabolic, or toxic etiology which required further testing, altered treatment, and improved the success rate of a client.

\section{References}

Arns, M. (2011). Personalized medicine in ADHD and depression: $A$ quest for treatment predictors (PhD dissertation). Utrecht University, Netherlands.

Arns, M., Swatzyna, R. J., Gunkelman, J., \& Olbrich, S. (2015). Sleep maintenance, spindling excessive beta and impulse control: an RDoC arousal and regulatory systems approach? Neuropsychiatric Electrophysiology, 2(1). http://dx.doi.org /10.1186/s40810-015-0005-9

Swatzyna, R. J., Kozlowski, G. P., \& Tarnow, J. D. (2015). Pharmaco-EEG. A study of individualized medicine in clinical practice. Clinical EEG and Neuroscience, 46(3), 192-196. http://dx.doi.org/10.1177/1550059414556120

Swatzyna, R. J., Tarnow, J. D., Tannous, J. D., Pillai, V., Schieszler, C., \& Kozlowski, G. P. (2014). EEG/QEEG Technology Identifies Neurobiomarkers Critical to Medication Selection and Treatment in Refractory Cases. Journal of Psychology \& Clinical Psychiatry, 1(7), 00046. http://dx.doi.org/10.15406 /jpcpy.2014.01.00046

The Effects of Misdiagnosed AttentionDeficit/Hyperactivity (ADHD) May Decrease Children's IQ, and The Efficacy of qEEG and Neurofeedback in the Assessment and Treatment of Misdiagnosed ADHD Children: A Clinical Case Series

Tanju Surmeli

Living Health Center for Research and Education, Sisli, Istanbul, Turkey

Background. In children with ADHD, some studies support the effect of stimulant medication on academic achievement and some do not. One problem may be the incorrect diagnosis of ADHD using subjective measures and another may be the inefficacy of treatment. If the problem is not addressed properly it may cause a decline in IQ scores as seen in our population. Neurofeedback was chosen as a treatment since there is evidence that neurofeedback in ADHD and LD has shown to be effective in this population and has also shown to be effective in improving IQ scores.

Methods. In this clinical case series, we analyzed the results of 21 medicated ADHD-diagnosed children and adolescents who did not show any substantial improvement and who had WISC-R results at least six months prior to coming to us. All the subjects were withdrawn from medication and tests were performed to determine the diagnosis and establish a baseline (qEEG Neurometric Analysis,
WISC-R, TOVA, and subjective questionnaires). These children were administered a qEEG-guided Neurofeedback protocol. The rationale being that NF would be effective in this population and another consideration was the parents' wishes of having a nonmedication alternative.

Results. At the end of the treatment all the tests were readministered and compared against baseline values. The results showed an increase in IQ scores with improvement in the all tests administered.

Conclusions. In this group, incorrect diagnoses, ineffective treatment, and the side effects of medication may cause a decline in the intellectual development of the children as observed by a decline in IQ scores. This decline was reversed with Neurofeedback treatment which not only showed improvement in objective measures (IQ scores) but on subjective measures also (rating scales). The implication for a clinical practice is that the overreliance on subjective measures may lead to an incorrect diagnosis and an ineffective treatment, having untoward effects on the child's intellectual development. Another finding of this study is that Neurofeedback treatment may be an effective treatment in this group of children.

\section{References}

Advisory Committee on Psychotropic Medications. (2004). Psychotropic Medication Utilization Parameters for Foster Children. Texas Department of State Health Services.

Aman, M. G., De Smedt, G., Derivan, A., Lyons, B., Findling, R. L., \& Risperidone Disruptive Behavior Study Group. (2002). Double-blind, placebo-controlled study of risperidone for the treatment of disruptive behaviors in children with subaverage intelligence. The American Journal of Psychiatry, 159(8), $\quad 1337-1346 . \quad$ http://dx.doi.org/10.1176 lappi.ajp.159.8.1337

American Psychiatric Association. (2013). Diagnostic and Statistical Manual of Mental Disorders (5th ed.). Washington, DC: Author.

Andersson, E. E., Bedics, B. K., \& Falkmer, T. (2011). Mild traumatic brain injuries: A 10-year follow-up. Journal of Rehabilitation Medicine, 43(4), 1-7. http://dx.doi.org/10.2340 /16501977-0666

Annegers, J. F., Grabow, J. D., Groover, R. V., Laws, E. R., Elveback, L. R., \& Kurland, L. T. (1980). Seizures after head trauma: A population study. Neurology, 30(7), 683 http://dx.doi.org/10.1212 /WNL.30.7.683

Gadow, K. D., Arnold, L. E., Molina, B. S. G., Findling, R. L., Bukstein, O. G., Brown, N. V., ... Aman, M. G. (2014). Risperidone Added to Parent Training and Stimulant Medication: Effects on Attention-Deficit/Hyperactivity Disorder, Oppositional Defiant Disorder, Conduct Disorder, and Peer Aggression. Child \& Adolescent Psychiatry, 53(9), 948-959. http://dx.doi.org/10.1016 /j.jaac.2014.05.008 
The Effectiveness of tDCS/tACS/tRNS and pEMF Stimulation on EEG Neurotherapy Performance

Nicholas Dogris

NeuroField, Inc., Bishop, California, USA

In this session, a discussion regarding neurostimulation technology will be conducted. The speaker will show how neurostimulation that is synchronized with EEG neurotherapy can enhance and facilitate regulation of the brain based on qEEG assessment and behavioral observational data. The limitations of EEG neurofeedback prevent adequate treatment outcomes on "tough cases" (such as autism) and decrease the credibility of the field in general when no treatment effects are observed. One of the main reasons for poor neurofeedback outcomes is associated with deregulation of the human connectome, or rich club. The human connectome has been strongly associated with the default mode network, and deregulation of this network can lead to poor communication between important network hubs that are responsible for regulating the brain. The speaker will discuss the current MRI BOLD research associated with physiological responses to $\mathrm{pEMF}$, tDCS/tACS/tRNS stimulation. In 2016 multiple studies were published that show how stimulation technology triggers calcium ion activation in the glia which gives rise to global neuroplasticity responses. This new data supports the use of neurostimulation methods as they can help to facilitate changes in absolute power, coherence, and phase towards the norm. The speaker will show and discuss qEEG and outcome data associated with clinical cases diagnosed with Autism, ADHD, depression, anxiety and Parkinson's disease. Each of the cases discussed with include a summary of patient information, diagnosis, history, and treatment plan. Each case will be presented with pre- and posttreatment EEG data. Behavioral changes will be discussed showing the effectiveness of EEG neurofeedback.

\section{References}

Bech, P., Lindberg, L., Straasø, B., \& Larsen, E. R. (2015). A 2 year follow-up study of patients participating in our transcranial pulsating electromagnetic fields augmentation in treatment-resistant depression. Neuropsychiatrica, 27(2), 119-125. http://dx.doi.org/10.1017/neu.2014.44

Cabral-Calderin, Y., Weinrich, C. A., Schmidt-Somoa, C., Poland, E., Dechent, P., Bahr, M., \& Wilke, M. (2016). Transcranial alternating current stimulation affects the BOLD signal in a frequency and task-dependent manner. Human Brain Mapping, 37(1), 94-121. http://dx.doi.org/10.1002/hbm.23016

Monai, H., Ohkura, M., Tanaka, M., Oe, Y., Konno, A., Hirai, H., ... Hirase, H. (2016). Calcium imaging reveals glial involvement in transcranial direct current stimulation-induced plasticity in mouse brain. Nature Communications, 7, 11100 http://dx.doi.org/10.1038/ncomms 11100

Schutter, D. J. L. G., \& Wischnewski, M. (2016). A meta-analytic study of exogenous oscillatory electric potentials in neuroenhancement. Neuropsychologia, 86, 110-118. http://dx.doi.org/10.1016/j.neuropsychologia.2016.04.011

Woods, A. J., Antal, A., Bikson, M., Boggio, P. S., Brunoni, A. R., Celnik, P., ... Nitsche, M. A. (2016). A technical guide to tDCS, and related non-invasive brain stimulation tools. Clinical Neurophysiology 127(2), 1031-1048. http://dx.doi.org /10.1016/j.clinph.2015.11.012

\author{
A Randomized Control Study of \\ Neurofeedback Training on Children with \\ Multiple Types of Trauma \\ Ainat Rogel ${ }^{1}$, Allyse Melville ${ }^{2}$, Michael Suvak ${ }^{3}$, Ed \\ Hamlin $^{4}$, Hilary Hodgdon ${ }^{1}$, Joseph Spinazzola ${ }^{1}$, and \\ Bessel van der Kolk \\ ${ }^{1}$ The Trauma Center at Justice Resource Institute, Brookline, \\ Massachusetts, USA \\ ${ }^{2}$ University of Connecticut, Storrs, Connecticut, USA \\ ${ }^{3}$ Suffolk University, Boston, Massachusetts, USA \\ ${ }^{4}$ Institute for Applied Neuroscience, Asheville, North Carolina, \\ USA
}

This research focuses on the effect of neurofeedback (NFB) on children with multiple types of childhood trauma. The Center for Disease Control (CDC) estimates that such traumas are arguably one of the most important public health challenges in the United States (van der Kolk, Roth, Pelcovitz, Sunday, \& Spinazzola, 2005). They have a negative impact on the mental and neurobiological functioning (Teicher \& Samson, 2016), which leads to a lower quality of life and creates a substantial financial burden for both the individuals affected and the healthcare industry (van der Kolk et al., 2005; Wang \& Holton, 2007). To date, there has been little research on the impact of NFB on multiple types of childhood trauma, although two recent studies on the impact of NFB on adults have shown that it significantly improves the condition of adults with chronic PTSD (Gapen et al., 2016; van der Kolk et al., 2016).

We present a randomized control design study of NFB on 37 children ages 6 to 13 who suffered from at least two types of trauma. The participants were randomly assigned into one of the two groups: Active $(n=20)$ and Control/waiting list $(n=17)$. The Active group received $24 \mathrm{NF}$ training sessions at T4P4 twice a week and underwent four periods of assessment: at baseline, midway through NFB, immediately posttraining, at a 1-month follow-up. The control group had assessments at equivalently spaced time points. Chi-square analyses were conducted to evaluate the impact on PTSD diagnoses (present/absent) according to K-SAD 
assessment. Piecewise growth curve analyses were run to explore differences in rates of change from baseline to posttreatment and from posttreatment to follow-up for both groups.

The results suggest that NFB significantly reduced the number of participants who met PTSD diagnosis criteria. Moreover, NFB significantly reduced, with effect sizes ranging from -0.49 (medium effect) to -0.96 (large effect), the symptoms for alexithymia, as measured in CAM assessment; cognitive and executive functioning, as measured in BRIEF assessment; and internalizing and externalizing behavior, as measured in CBCL assessment.

This study suggests that NFB is an effective treatment for improving the condition of children with multiple types of trauma. Moreover, most care givers reported that the children were resistant to other therapies (note that resistant to other therapies was not an inclusion criteria).

We therefore recommend further study with a larger number of participants, personalized protocol and more NFB sessions.

\section{References}

Gapen, M., van der Kolk, B. A., Hamlin, E., Hirshberg, L., Suvak, M., \& Spinazzola, J. A. (2016). Pilot Study of Neurofeedback for Chronic PTSD. Applied Psychophysiology and Biofeedback, 41(3), 251-261. http://dx.doi.org/10.1007 /s10484-015-9326-5

Teicher, M. H., \& Samson, J. A. (2016). Annual Research Review: Enduring neurobiological effects of childhood abuse and neglect. Journal of Child Psychology and Psychiatry, 57(3), 241-266. http://dx.doi.org/10.1111/jcpp.12507

van der Kolk, B. A., Hodgdon, H., Gapen, M., Musicaro, R., Suvak, M. K., Hamlin, E., \& Spinazzola, J. (2016). A randomized controlled study of neurofeedback for chronic PTSD. PLoS ONE 11(12), e0166752. http://dx.doi.org /10.1371/journal.pone.0166752

van der Kolk, B. A., Roth, A., Pelcovitz, D., Sunday, S., \& Spinazzola, J. (2005). Disorders of Extreme Stress: The Empirical Foundation of a Complex Adaptation to Trauma. Journal of Traumatic Stress, 18(5), 389-399. http://dx.doi.org /10.1002/jts.20047

Wang, C.-T., \& Holton, J. (2007). Total Estimated Cost of Child Abuse and Neglect in the United States. Economic Impact Study. Chicago, IL: Prevent Child Abuse America.

\section{Impact of Developmental Trauma on Brain}

Function and Connectivity

Carl Armes and Robert Coben

Integrated Neuroscience Services, Fayetteville, Arkansas, USA

The effect of trauma upon anyone can have a longlasting impact on their brain. For children, trauma can have a permanent impact on their developing brain, yet they may never meet the criteria for PTSD
(Teicher, Andersen, Polcari, Anderson, \& Navalta, 2002). For children however, traumatic stress can come from multiple and/or chronic and prolonged, developmentally adverse traumatic events during early childhood development (van der Kolk, 2005). This has led to a new classification, Developmental Trauma (DT). Areas of the brain involved in the stress response include the amygdala, hippocampus, and prefrontal cortex, which also play a role in memory (Bremner, 2006). Research by Sapolsky found stress can alter plasticity of the limbic system, not only affecting hormone secretion, but also how the hippocampus and amygdala work together to form memories about the stressor (2003). Comparing maltreated groups to control groups, Teicher, Samson, Anderson, and Ohashi (2016) also found connectivity issues as well in the left anterior cingulate, right occipital pole, left temporal pole, and right medial frontal gyrus. Regions of decreased connectivity were found in areas important to emotional regulation, attention, and social cognition, while areas with increased connectivity seemed to occur in areas of selfregulation (Teicher, Samson, Anderson, \& Ohashi, 2016).

EEG/qEEG research on developmental trauma is quite scant. One study found that abused children had higher left hemisphere coherence and a reversed asymmetry as well as a slower rate of decay of left hemisphere coherence over electrode distance suggesting deficit in left cortical differentiation (Ito, Teicher, Glod, \& Ackerman, 1998). Our study aims to study the impact of repeated developmental trauma on brain function and connectivity. We hypothesize adults that experienced DT will show significantly different findings than those that did not have such a history. Further, we anticipate that susceptible regions may include those near the anterior cingulate left frontal temporal and limbic regions and right posterior regions involved in social engagement.

We are in the midst of collecting QEEG data (19 and 64 channels) on 30 survivors of DT and a comparison group. The groups will be compared for differences in EEG power, coherence, and connectivity. Using EEGLAB and MVGC (multivariate granger causality toolbox) we will measure scalp and source measures for comparison as indicated above. Source comparisons will be made insuring finer spatial localization of the network components while minimizing signal processing confounds produced by broad volume conduction from neural sources to the scalp electrodes (Coben, Mohammad-Rezazadeh, \& 
Cannon, 2014). Source-derived connectivity measures including Granger Causality and partial directed coherence will be applied. Group-based comparisons of these metrics will be displayed and case examples will be used as well for illustrative purposes. The implications of these findings for understanding DT and its treatment will be discussed.

\section{References}

Bremner, J. D. (2006) Traumatic stress: Effects on the brain. Dialogues in Clinical Neuroscience, 8(4), 445-461.

Coben, R., Mohammad-Rezazadeh, I., \& Cannon, R. L. (2014) Using quantitative and analytic EEG methods in the understanding of connectivity in autism spectrum disorders: A theory of mixed over- and under-connectivity. Frontiers in Human Neuroscience, 8, 45. http://dx.doi.org/10.3389 /fnhum.2014.00045

Coben, R., Mohammad-Rezazadeh, I., Frohlich, J., Jurgiel, J., \& Michelini, G. (2017). Imaging brain connectivity in autism spectrum disorder. In M. F. Casanova, A. El-Baz, \& J. S. Suri (Eds.), Autism Imaging and Devices. Boca Raton, FL: CRC Press.

Ito, Y., Teicher, M. H., Glod, C. A., \& Ackerman, E. (1998) Preliminary evidence for aberrant cortical development in abused children: A quantitative EEG study. The Journal of Neuropsychiatry and Clinical Neurosciences, 10(3), 298-307. http://dx.doi.org/10.1176/jnp.10.3.298

Sapolsky, R. M. (2003). Stress and plasticity in the limbic system. Neurochemical Research, 28(11), 1735-1742. http://dx.doi.org/10.1023/A:1026021307833

Teicher, M. H., Andersen, S. L., Polcari, A., Anderson, C. M., \& Navalta, C. P. (2002). Developmental neurobiology of childhood stress and trauma. Psychiatric Clinics of North America, 25(2), 397-426. http://dx.doi.org/10.1016/S0193$953 \times(01) 00003-X$

Teicher, M. H., Samson, J. A., Anderson, C. M., \& Ohashi, K. (2016). The effects of childhood maltreatment on brain structure, function and connectivity. Nature Reviews Neuroscience, 17, 652-666. http://dx.doi.org/10.1038 /nrn.2016.111

van der Kolk, B. A. (2005). Developmental Trauma Disorder: Toward a rational diagnosis for children with complex trauma histories. Psychiatric Annals, 35(5), 401-408. http://dx.doi.org/10.3928/00485713-20050501-06

\section{Alpha Phase Synchrony Based Neurofeedback for Chronic Back Pain Louis Mayaud ${ }^{1}$, Quentin Barthelemy ${ }^{1}$, Yannick Delpierre ${ }^{2}$, Helene $W u^{2}$, Patrick Favennec ${ }^{3}$, Michel Ritz $^{2}$, and Marco Congedo ${ }^{4}$ \\ ${ }^{1}$ Mensia Technologies, Paris, Ile-de-France, France \\ ${ }^{2}$ Center of the Ark, Saint-Saturnin, Pays de la Loire, France \\ ${ }^{3}$ Covéa Technologies, Paris, Ile-de-France, France \\ ${ }^{4}$ Grenoble Institute of Technology, Grenoble, Auvergne-Rhone- Alpes, France}

Chronic back pain is a well spread phenomenon affecting as many as $2 \%$ of the French population with a conservative estimate of $1.5 \mathrm{~m}$ chronic patients just in France for whom all therapeutic options have failed. The direct cost for the payer is estimated to a yearly $€ 1.6 \mathrm{~b}$ for France, turning a relatively small population into a public health issue. Neurofeedback (NFB) is a self-paced brain neuromodulation technique that represents one's brain activity in real-time using auditory or visual modulations, on which the subject can exerts voluntary control, or which is used to "condition" certain neural mechanisms. Brain activity is captured using an electroencephalographic (EEG) device.

The goal of this pilot study is to evaluate the efficiency of alpha synchrony based NFB, applied to chronic low back pain patients $(n=16)$ with whom all available therapeutic options have failed. The intervention investigated is twenty 30 -min-long alpha synchrony neurofeedback session using an EEG cap of 21 electrodes. It is an open-label study with no control group. Patients were included after failing all other therapeutic options including a 2-week-long pluri-disciplinary approach dispensed at a tertiary hospital.

First, progression of clinical scores (before and after intervention) such as pain, anxiety, depression, and quality of life are shown to have been reduced by more than $25 \%$ in most of patients. Likewise, electromyographic (EMG) signals show a statistically significant increase of the median frequency, associated to a gain of motor units of bigger diameters and more dynamic, the opposite that one can observe during muscle fatigue. Finally, alpha synchrony neuromarkers extracted from EEG signals at each session show progressions along blocks and sessions. More interestingly, the slope progression over sessions is correlated with the decrease of clinical score, which indicates specificity of the trained neuromarker with respect to clinical outcomes.

Future works involves the analysis of 6- and 12month clinical and EEG follow-up data to investigate the long-term efficacy of neurofeedback. The results of this pilot project would ultimately be the clinical and technical foundations of a high-impact RCT to limit use of analgesics and nonsteroidal antiinflammatory drugs (NSAID) and promote quick return to work for these patients.

\section{References}

Arns, M., de Ridder, S., Strehl, U., Breteler, M., \& Coenen, A. (2009). Efficacy of neurofeedback treatment in ADHD: The effects on inattention, impulsivity and hyperactivity: A metaanalysis. Clinical EEG and Neuroscience, 40(3), 180-189, 2009, http://dx.doi.org/10.1177/155005940904000311 
McKnight, J. T., \& Fehmi, L. G. (2001). Attention and neurofeedback synchrony training: Clinical results and their significance. Journal of Neurotherapy, 5(1-2), 45-61, http://dx.doi.org/10.1300/J184v05n01_05

Received: November 16, 2017

Accepted: November 16, 2017

Published: December 8, 2017 